\title{
Study on a New Operational Mode of Economic Operation of Islanded Microgrids Using Electric Springs
}

\author{
Zhiyu Zhao ${ }^{1}$, Keyou Wang ${ }^{1}$, Guojie Li ${ }^{1}$ Xiuchen Jiang ${ }^{1}$, Yin Zhang ${ }^{2}$ \\ ${ }^{1}$ Department of Electrical Engineering, Shanghai Jiao Tong University, 200240, Shanghai, China \\ ${ }^{2}$ Shanghai Electric Power Technical Research Institute of State Grid, 200247, Shanghai, China
}

\begin{abstract}
With the increasing penetration of intermittent renewable energy sources (RESs) into microgrids, the original operation mode of power generation determined by load demand faces severe challenges due to the uncertainties of the RESs power output. The electric springs(ESs), as an emerging technology has been verified to be effective in enabling load demand to follow power generation and stabilizing fluctuation of RESs output. This paper presents a new mode of economic operation for island microgrids including non-critical loads with embedded electric springs. Its connotation includes that i) the capacity of energy storage can be reduced through the interaction of the energy storage system (ESS) and the electric springs, ii) the electric springs reduce the stress of peak load regulation and operational cost and iii) the demand of microgrids system for ramping ability of generation units is reduced with the buffer of the electric springs. Numerical results show that the coordinated operation between electric springs and energy storage system of microgrids can bring down the investment cost for the ESS and short-term operational cost in the aspect of economic dispatch, reducing requirements for the capacity and ramp ability of the energy storage system in microgrids. Energy buffering can be achieved with lower cost and the load demand can follow power generation in the new operational mode of islanded microgrids using electric springs.
\end{abstract}

\section{Introduction}

Traditionally, load demand determines power generation in operational mode of power system [1]. With the increasing penetration of intermittent renewable energy sources (RESs), just like photovoltaic and wind power into microgrids year by year, it is difficult to accurately predict the total amount of power generation and achieve real-time power balance between load demand and power generation. The following problems, including voltage fluctuations and frequency flicker [2], are more and more obvious. Large grids have some self-regulation capability for voltage fluctuations, while small and islanded microgrids have poor regulation capabilities with the risk of damage to electrical equipment. It has been proposed that using battery, superconducting or high-speed flywheel energy storage device to offset the difference between power generation and load demand is one of the effective ways to solve this problem. However, the energy storage system (ESS) such as batteries are expensive with higher cost and the used batteries are the main source of contaminants. The operational mode of microgrids is moving to "power generation determine load demand". Demand energy management research has arisen, including load scheduling [3], differentiated electricity prices and so on. However, most of those methods are suitable for hour-interval load requirements management, not for real-time energy balance. The new technology of the electric springs (ESs) has recently been proposed for load-side energy management with a large absorption of intermittent renewable power, instantaneously transferring energy fluctuations to noncritical loads and automatically adjusting the power consumption of non-critical loads, with automatic balance between power generation and consumption.

This paper proposed the new mode of economic operation for island microgrids including non-critical loads with embedded electric springs. The capacity of energy storage can be reduced with the electric springs [4]. Due to the interaction of the ESS and the ESs, the timely adjustment of the power consumption of noncritical loads can reduce the charge and discharge power. The double layer optimal dispatch algorithm for islanded microgrid with ESs has been proven to be effective to determine the minimum ESS capacity meeting a certain scenario. When the peak load comes, the electric springs can reduce the power system's demand for the ability of peak load regulation, so as to enhance short-term economic benefits. The demand of microgrids for ramping ability of the ESS or other generation units is reduced with the buffer of the electric springs with lower cost for design and manufacture of generation units. The coordinated operation between the ES and ESS of microgrid can bring down the investment cost of the ESS and short-term operational cost in the aspect of economic 
dispatch. Energy buffering can be achieved for lower cost and the load demand can track power generation in the new operational mode of islanded microgrids using electric springs. The excellent function of the energy buffer of the ESs will provide a novel access for the construction of the future microgrids. Three advantages of ESs mentioned above have been demonstrated in the model of optimal dispatch in a hypothetical microgrid example.

\section{Basic principles of electric springs in islanded microgrids}

This hypothetical case is performed by an islanded microgrid containing wind power, ESS, diesel generators and two kinds of load. Critical loads require higher power quality supplies and do not allow for relatively large voltage fluctuations. Non-critical loads, such as electric heaters, have a wide voltage range of operation. The voltage of the ESs will changes according to the real-time power balance. Normally, power generation will follow the load demand without the ES.

Typical, a three-phase electric spring connect in series with a non-critical load. The series combination connects in parallel with critical load. The ES itself does not participate in the regulation of the active power of the microgrid system, but changes the power consumed by changing the voltage of the non-critical load.

There are two kinds of operational mode of the ES, including capacitive mode and inductive mode. The capacitive mode matches with different loads, shown in Fig.1. The magnitude of the voltage of the ES determines the angle of the voltage vector between the critical load and the non-critical load, and the impedance factor angle of the non-critical load also affects it [5].

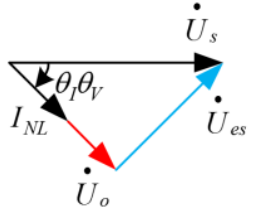

(a)

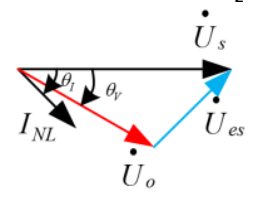

(b)

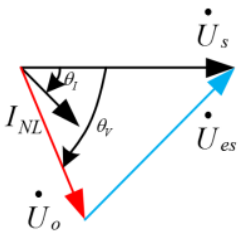

(c)
Figure 1. The Capacitive Modes of ES connected with (a) Resistive load. (b) Resistive-inductive load. (c) Resistivecapacitive load.

Where $I_{N L}$ is current of non-critical load. The vector formulations about voltage and power between the power spring and the load are as follows:

$$
\begin{gathered}
\dot{U}_{s}=\dot{U}_{o}+\dot{U}_{e s} \\
P_{c l}=3 \times \operatorname{re}\left(\dot{U}_{s} \times\left(\frac{\dot{U}_{s}}{Z_{c l}}\right)^{*}\right) \\
P_{n l}=3 \times \operatorname{re}\left(\dot{U}_{o} \times\left(\frac{\dot{U}_{o}}{Z_{n l}}\right)^{*}\right) \\
P=P_{c l}+P_{n l}
\end{gathered}
$$

Where $\dot{U}_{s}$ and $\dot{U}_{o}$ denotes the voltage of critical and non-critical load of single phase. And $\dot{U}_{e s}$ denotes the voltage of the ES of single phase. The voltage of noncritical load moves between 0.8 and 1.0p.u. with the rated voltage 0.9 p.u., for peak and valley load regulation. The voltage of critical load will be maintained at 1.0 p.u. if without a sudden emergency.

\section{Double layers optimal dispatch algorithm for islanded microgrid with electric springs}

The economic dispatch of microgrid is divided into dayahead and intraday dispatch. Intraday dispatch requires for short-term or ultra-short-term load forecasting data. The energy buffer of the ES can be applied to day-ahead dispatch and real-time adjustment of intraday dispatch.

Double layers optimal dispatch algorithm for islanded microgrid with ESs can be more suitable for real-time dispatch. The 1st layer optimal dispatch is to minimize the operational cost within a day and meanwhile constrained by definite operating constraints of islanded microgrid. Based on the solution of the 1 st layer, the 2 nd layer optimal dispatch can be used to obtain the minimum capacity requirement of ESS that can meet the current micro-grid, the least ramping ability requirement of the generator units and the obvious effect of lowering the pressure of peak load regulation in microgrid with the buffering of the electric springs.

\subsection{The 1st Layer- Optimal Dispatch Formulation of the Islanded Microgrid}

The 1st layer optimal dispatch algorithm is similar to the traditional economical dispatch of microgrid [6]. This optimization objective can be formulated as generation costs function of diesel generator:

$$
\min \left\{\sum_{t=1}^{N}\left[\lambda D_{t}\right]\right\}
$$

Where $N$ denotes the number of dispatch intervals within a day. $\lambda$ and $D_{t}$ are the generation unit price and output power of diesel generator in the $t$ th interval.

Considering power output, capacity and actual scenario of this microgrid, multiple definite operating constraints are as follows:

$$
\begin{gathered}
0 \leq W_{t} \leq W_{\max } \\
\left|W_{t+1}-W_{t}\right| \leq R_{w \max } \\
-S_{c \max } \leq S_{t} \leq S_{d \max } \\
\left|S_{t+1}-S_{t}\right| \leq R_{s \max } \\
0 \leq D_{t} \leq D_{d \max }-D_{r} \\
\left|D_{t+1}-D_{t}\right| \leq R_{d \max } \\
W_{t}+S_{t}+D_{t}=P_{c l-t}+0.81 P_{n l-t}
\end{gathered}
$$

Where Equation (6)-(7) demonstrate the power output upper limits constraints and ramping constraints of wind power. Equation (8)-(11) are similar constraints of ESS 
and diesel generators. $D_{r}$ is spare capacity of diesel generator groups to prevent the extreme scenarios of islanded microgrid. In general, $D_{r}=0.1 D_{d \max }$ Equation (12) represents the real-time power balance in the microgrid considering critical and non-critical loads.

When the ESS is discharged, $S(t) \geq 0$ and the state of charge (SOC) can be expressed as:

$$
S O C(t)=(1-\eta) S O C(t-1)-\frac{S(t) \Delta t}{\eta_{d} E_{b}}
$$

When the ESS is charged, $S(t) \leq 0$ and

$$
\operatorname{SOC}(t)=(1-\eta) \operatorname{SOC}(t-1)-\frac{S(t) \eta_{c} \Delta t}{E_{b}}
$$

The SOC of ESS changes within safe operating range. And in order to ensure the ESS's ability to adjust of the next day, the change in SOC within a day shall not exceed a proportion of the maximum capacity:

$$
\begin{gathered}
S O C_{\min } \leq S O C_{t} \leq S O C_{\text {max }} \\
\left|S O C_{N}-S O C_{1}\right| \leq \delta
\end{gathered}
$$

The solution of the $1^{\text {st }}$ layer optimal dispatch can be used to determine the smaller operational cost, regardless of the electric springs.

\subsection{The 2nd Layer- Collaborative Optimization based on Interaction of ESS and ES}

The $2^{\text {nd }}$ layer optimal dispatch focuses on the interaction of ESS and ES. The ES can adjust the non-critical load demand, thus reducing current and power of charge and discharge of the ESS based on the same voltage. This optimization objective can be formulated as the minimum of total power of charge and discharge of ESS:

$$
\min \left\{\sum_{t=1}^{N}\left|S_{t}\right|\right\}
$$

If the interaction of ESS and ESs is permitted, the constraints of power balance can be amended and strengthened as:

$$
S_{1-t}+0.81 P_{n l-t}=S_{2-t}+\left(0.81+k_{t}\right) P_{n l-t}
$$

Where $S_{1-t}$ and $S_{2-t}$ are the solutions of ESS from the $1^{\text {st }}$ and $2^{\text {nd }}$ layer optimal dispatch. The constraint of noncritical load power regulation coefficients $k_{t}$ is as follows:

$$
0.64 \leq 0.81+k_{t} \leq 1
$$

If the $2^{\text {nd }}$ layer optimal dispatch focuses on pressure relief of peak load regulation, the interaction of diesel generators and ES will lower the cost of diesel generators or other dispatchable units during peak load period. And Equation (19) can be modified as:

$$
\begin{aligned}
D_{1-t}+0.81 P_{n l-t} & =D_{2-t}+\left(0.81+k_{t}\right) P_{n l-t} \\
t & \in \Omega
\end{aligned}
$$

Where $\Omega$ is the periods set that requires the peak load regulation, determined from the $1^{\text {st }}$ layer optimal dispatch. $D_{1-t}$ is also the solution of diesel generator from the $1^{\text {st }}$ layer.

The $2^{\text {nd }}$ layer optimal dispatch demonstrates the benefits of the energy buffering of the ES, with the applications of reducing the cost of investment of the ESS and pressure peak load regulation. Double layers optimal dispatch algorithm with ESs can be suitably used in economical dispatch in the future islanded microgrid on the new operational mode.

The mathematical nature of double layers optimal dispatch algorithm for islanded microgrid with the ES can be attributed to the second-order cone programming (SOCP) problem and can be effectively solved.

\section{Numerical results}

This paper takes the microgrid of region of Beijing Olympic Winter Games as the hypothetical case. The formulations of double layers optimal dispatch algorithm for islanded microgrid with the ES are programmed in MATLAB and the summarized SOCP model is solved using Gurobi [7]. The simulations are carried out on an Intel Core i3-4130 CPU running at $3.40 \mathrm{GHz}$ with $8 \mathrm{~GB}$ of RAM.

The microgrid is expected to be configured with wind power capacity of $2 \mathrm{MW}$, energy storage capacity of $17.3 \mathrm{MW} \cdot h$ and diesel generation capacity of $600 \mathrm{~kW}$. Part of parameters of the output units in the microgrid are shown in Table 1.

Table 1 Parameters of the Islanded Microgrid

\begin{tabular}{|c|c|c|c|}
\hline Name & Value & Name & Value \\
\hline$S_{c \max }$ & $800 \mathrm{~kW}$ & $S_{d \max }$ & $600 \mathrm{~kW}$ \\
\hline$R_{s \max }$ & $400 \mathrm{~kW}$ & $R_{w \max }$ & $700 \mathrm{~kW}$ \\
\hline$\eta$ & 0.00001 & $R_{d \max }$ & $560 \mathrm{~kW}$ \\
\hline$\eta_{d}$ & 0.9 & $E_{b}$ & $17.3 M W \cdot h$ \\
\hline$S O C_{\min }$ & 0.2 & $S O C_{\max }$ & 0.9 \\
\hline$\delta$ & 0.05 & $\eta_{c}$ & 0.85 \\
\hline$\lambda$ & $3.2 \mathrm{yuan} /(\mathrm{kW} \cdot \mathrm{h})$ & $\mathrm{N}$ & 48 \\
\hline
\end{tabular}

The forecasting curve of wind power output and load of the region is shown in Fig 2. The total load in this microgrid consists of critical and non-critical load. The ratio of the critical load to the total load is different at different dispatch interval of one day. The ESS will firstly charge, then discharge and finally charge because of the constraint of $\left|S O C_{N}-S O C_{1}\right| \leq \delta$ with the benefits of sustainability in the normal operational mode of the islanded microgrid.

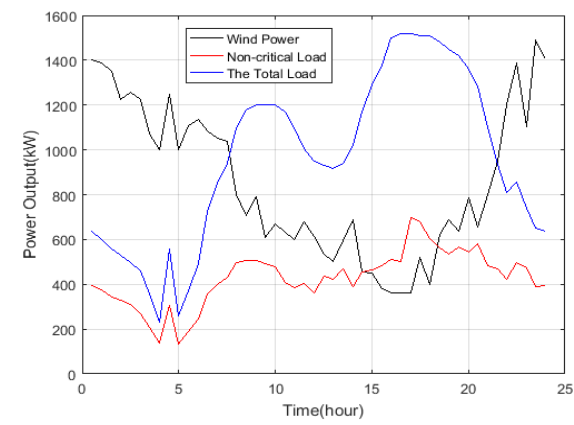

Figure 2. Forecasting Curve of Wind Power Output, Noncritical Load and Total Load 
The solution of traditional optimization dispatch is shown in Fig. 3 with the lowest cost based on the forecasting RES and constraints of generation units regardless of the ES. The ESS still has the capacity margin when non-peak load. During the peak load from 15:00 to 20:00, the ESS reaches the maximum output and diesel generators are put into operation from Fig.6. The RESs output the maximum power as soon as possible within the operational constraints.

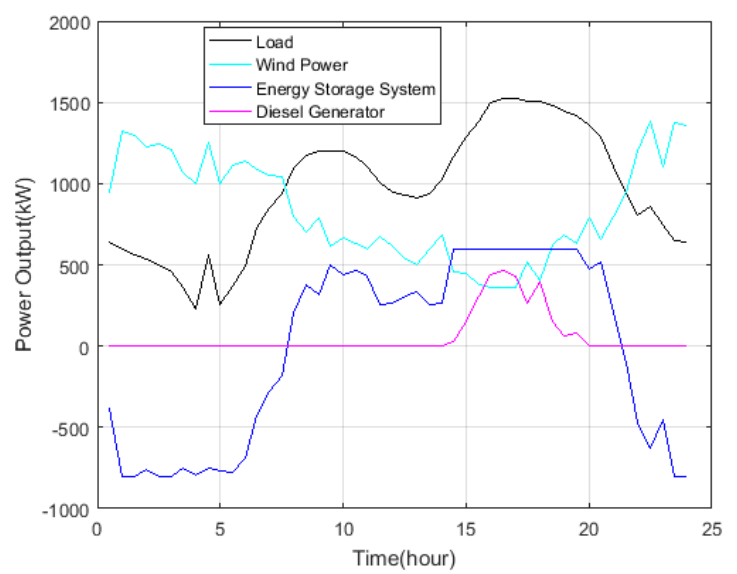

Figure 3. Solution Curve for Optimization Dispatch without the ES

\subsection{Reduction and Determination of the Capacity of ESS}

The SOC curve of ESS can be obtained when the capacity of the ESS changes in the range of $\left[0.6 E_{b}, E_{b}\right]$ as Fig.4 and it can be concluded that one day's operational cost of islanded microgrid will be maintained at 4423.22 yuan if the capacity of ESS is above $0.6 E_{b}$ after many tests. But cost will rise and efficiency will decline (It is unnecessary to discharge firstly, then charge when the wind power is rich in the red cycle) if the capacity of the ESS below the threshold of $0.6 E_{b}\left(0.599 E_{b}\right.$ more precisely).

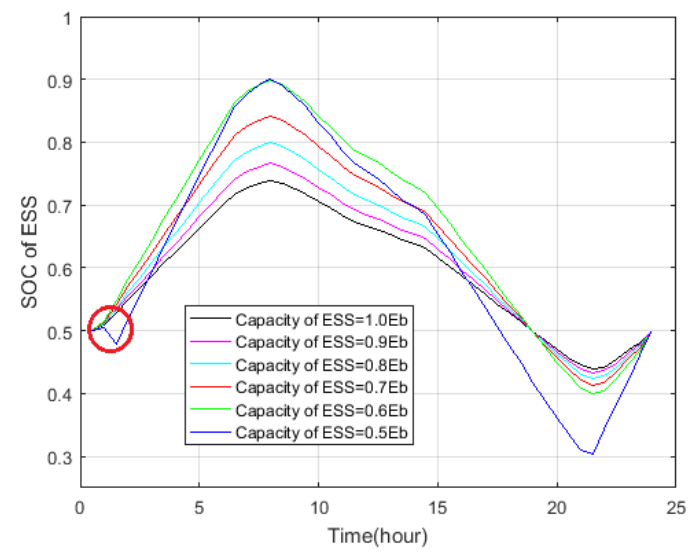

Figure 4. SOC Curve when the Capacity when the ESS Changes

If the 2nd layer collaborative optimization based on interaction of ESS and ES is enabled with the $0.6 E_{b}$ capacity of the ESS, the charge and discharge power of the ESS is reduced, and the variation range of the SOC is smaller (that is to say, a part of the energy storage capacity is surplus if not considering the operational margin of microgrid.), shown in Fig.5-6.

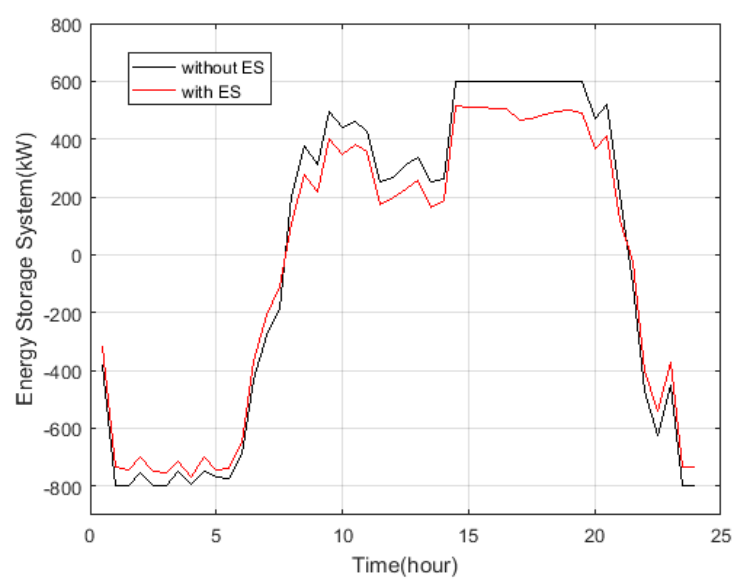

Figure 5. Charge and Discharge Power Curve of the ESS with and without the ES

The further numerical results demonstrate that the operational cost of one day can still be maintained at 4423.22 yuan when the capacity of the ESS drops to with the help of ES. That is to say, the capacity of the ESS decreased by $7.8 \%$. However, the operational cost of one day has soared to $5488.89 y$ yan without the ES when the capacity of the ESS is adopted.

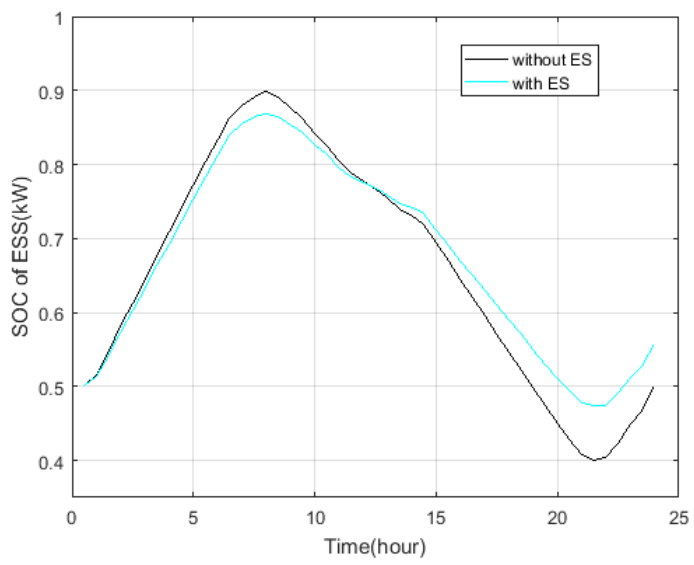

Figure 6. SOC Curve of the ESS with and without the ES

\subsection{Pressure Mitigation of Peak Load Regulation}

When the electric springs are used for peak load regulation of microgrids, the mere participation of collaborative optimization with generation units that generate short-term costs, like diesel generators, directly reduces operational costs of one day, mitigating pressure of peak load regulation simultaneously. The economical dispatch results with ES only applied in peak load regulation are shown in Fig.7. 
The function of the electric spring under the peak load can be summarized as the electric spring for passive load regulation only for a short time.

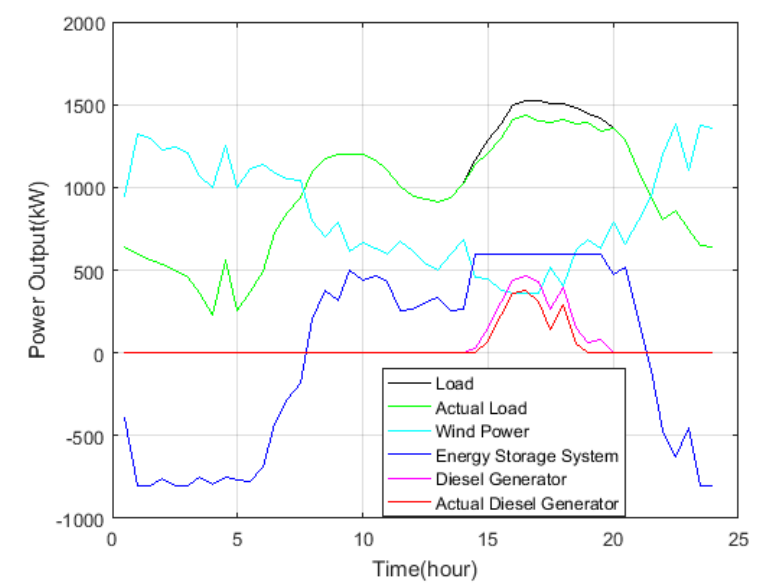

Figure 7. Solution Curve for Optimization Dispatch with ES for Peak Load Regulation

\subsection{Reduction and Determination of Requirements for Ramping Ability}

The ES can be used to reduce the requirements of ramping ability of generator in islanded microgrid. When the ramping capacity $R_{s \max }$ of the ESS drops from $400 \mathrm{~kW}$ to $352 \mathrm{~kW}$ without ES, the operational cost in one day will be maintained at 4423.22 yuan without the ES. When the ramping capacity of the ESS is below $352 \mathrm{~kW}$, the operational cost in one day of microgrid will rise. The optimal dispatch result of microgrid with the ES is shown in Fig.8. Numerical simulation results show that the operational cost in one day will not yet increase when the ramping capacity of ESS is reduced to $265 \mathrm{~kW}$ with the help of ES, that is to say, electric spring makes ramping capacity decreased by $25 \%$ maximum.

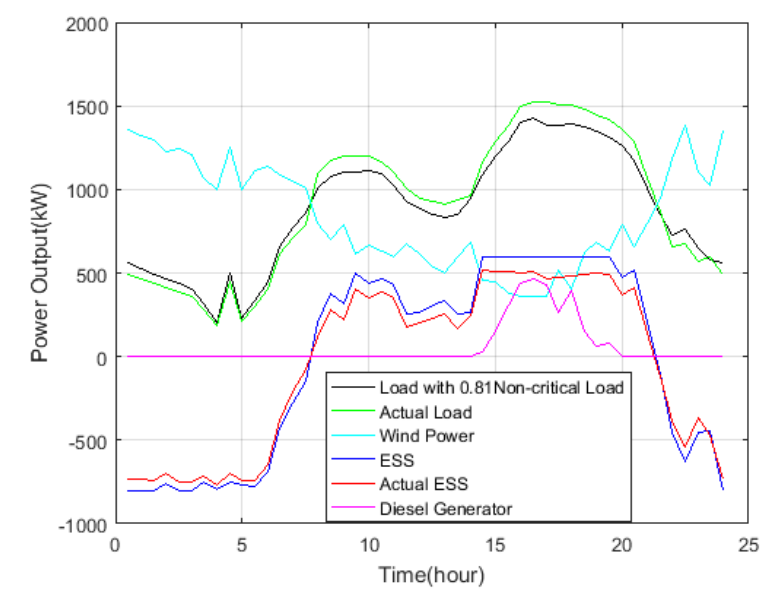

Figure 8. Optimal Dispatch Result of Microgrid with ES

If the power springs can be widely installed in the microgrid, the need for ramping ability of the generator units will be greatly reduced, thereby reducing the manufacturing costs. The function of the electric spring cooperated with the ESS can be summarized as the electric spring for interactive operation for a long time.

\section{Conclusion}

The study on a new mode of economical operation of islanded microgrids using electric springs has been pushed forward. The double layers optimal dispatch algorithm for islanded microgrids using ES is proposed in this paper. The mode of "load demand following power generation" has been effectively verified into operation for island microgrids including non-critical loads with embedded electric springs with three important benefits including that i) the reduction of capacity of ESS through the interaction of the ESS and the ES, ii) pressure mitigation when peak load regulation using the electric springs with lower cost and iii) reduction of requirements for ramping ability of generation units with the energy buffering of the ES. Numerical results, obtained on a hypothetical case, show that the coordinated operation between electric springs and energy storage system of microgrids can bring down the investment cost in capacity and ramping ability of the ESS and short-term operational cost in one day. The electric springs can be reliably used in future microgrids to change the traditional operating modes.

\section{Acknowledgment}

This work was supported by State Grid Corporation of China Science \& Technology Project on Principle, Design and Control Technology of New Operation Mode of Microgrid Based on Electric Spring.

\section{References}

1. D. Westermann and A. John, "Demand matching wind power generation with wide-area measurement and demand-side management,"IEEE Trans. Energy Convers., vol. 22, no. 1, pp. 145-149, 2007.

2. Cheng $\mathrm{M}, \mathrm{Zhu} \mathrm{Y}$. The state of the art of wind energy conversion systems and technologies:A review $[\mathrm{J}]$. Energy Conversion and Management, 2014(88):332-347.

3. A. Mohsenian-Rad, V. W. S. Wong, J. Jatskevich, R. Schober, and A.Leon-Garcia, "Autonomous demandside management based on gametheoretic energy consumption scheduling for the future smart grid,"IEEE Trans. Smart Grid, vol. 1, no. 3 , pp. 320-331, 2011.

4. C. K. Lee, and S. Y. Hui, "Reduction of Energy Storage Requirements in Future Smart Grid Using Electric Springs," IEEE Trans. Smart Grid, vol. 4, no. 3, pp. 1282-1288, 2013.

5. N. R. Chaudhuri, C. K. Lee, B. Chaudhuri et al., "Dynamic Modeling of Electric Springs," IEEE Trans. Smart Grid, vol. 5, no. 5, pp. 2450-2458, 2014.

6. S. Conti et al, "Optimal dispatching of distributed generators and storage systems for MV islanded microgrids," IEEE Trans. Power Del., vol. 27, no. 3, pp. 1243-1251, Jul. 2012

7. Gurobi Optimizer 7.5, Jul. 2017 [Online]. Available: http://www.gurobi.com 